\title{
Salt-Wasting 21-Hydroxylase Deficiency
}

National Cancer Institute

\section{Source}

National Cancer Institute. Salt-Wasting 21-Hydroxylase Deficiency. NCI Thesaurus. Code C131134.

A classic form of congenital adrenal hyperplasia characterized by complete absence of 21-hydroxylase activity resulting in deficiency of glucocorticoids and mineralocorticoids accompanied by androgen excess causing virilization in female infants. Mineralocorticoid deficiency results in renal salt-wasting, and if untreated, hyponatremia, hyperkalemia, and shock. 